\title{
Analytic Model of the Collision Resolution Protocol with Voice/Data in Mobile Networks
}

\author{
Dong Chun Lee \\ Dept. of Computer Science, Howon Univ. Korea \\ ldch@sunny.ac.kr
}

\begin{abstract}
We propose analytic model of the delay of the Slotted ALOHA protocol with Binary Exponential Backoff (BEB) as a collision resolution algorithm in mobile multimedia networks. We verify the proposed analytic model using the computer simulation. In numerical results, our proposed method show correct analytic model and has better performance results than previous methods in mobile multimedia networks.
\end{abstract}

\section{Introduction}

In Slotted ALOHA system, each frame is divided into small slots and each Mobile Terminal (MT) contends for the slot to transmit its packets at the beginning of each frame. If two more than MTs contend for the same slot, then a collision occurs and none of them can transmit their packets. The colliding packets are queued and retry after a random delay. The way to resolve the collision is called the collision resolution protocol. One of the widely used collision resolution protocol is the BEB algorithm, forms of which are included in W- LAN standards: Whenever a node's call is involved in a collision, it selects one of the next $2^{n}$ frames with equal probabilities, where $n$ is the number of collisions that the call has ever experienced, and attempts the retransmission.

Delay distributions of slotted ALOHA and CSMA are derived in Yang and Yum [2] under three retransmission policies. They found the conditions for achieving finite delay mean and variance under the BEB. Their assumption, however, that the combination of new and retransmitted packet arrivals is a Poisson process is not valid because the stream of the retransmitted packets depends on the arrivals of new packets. This dependency makes the Poisson assumption invalid. Chatzimisios and Boucouvalas [4] presented an analytic model to compute the throughput of the IEEE 802.11 protocol for wireless LAN and examined the behavior of the Exponential Backoff (EB) algorithm used in 802.11. They assumed that the collision probability of a transmitted frame is independent of the number of retransmissions. As we will show later in this paper, however, this probability is a function of the number of competing stations and also depends upon the number of retransmissions that this station has ever experienced. Kwak et al. [4] gave new analytical results for the performance of the EB algorithm. Especially, they derived the analytical expression for the saturation throughput and expected access delay of a packet for a given number of nodes. Their EB model, however, is assumed that the packet can retransmit infinitely many times.

X. Zhou et al. (Eds.): EUC Workshops 2006, LNCS 4097, pp. $953-961,2006$.

(C) IFIP International Federation for Information Processing 2006 
Stability is another issue on BEB algorithm and there are many methods dealing with this. As pointed in Kwak et al. [4], however, these studies show contradictory results because some of them do not represent the real system and they adopt different definitions of stability used in the analyses. The dispute is still going on so we do not focus on this topic but on the analytic model to analyze the performance of the BEB algorithm.

In this paper we propose a new analytical model to find the performance measures to evaluate the system which adopts BEB algorithm, including the throughput, expected medium access delay and transmission delay.

\section{System Model}

Fig. 1 shows the procedure considered the access delay and transmission delay in any cell.

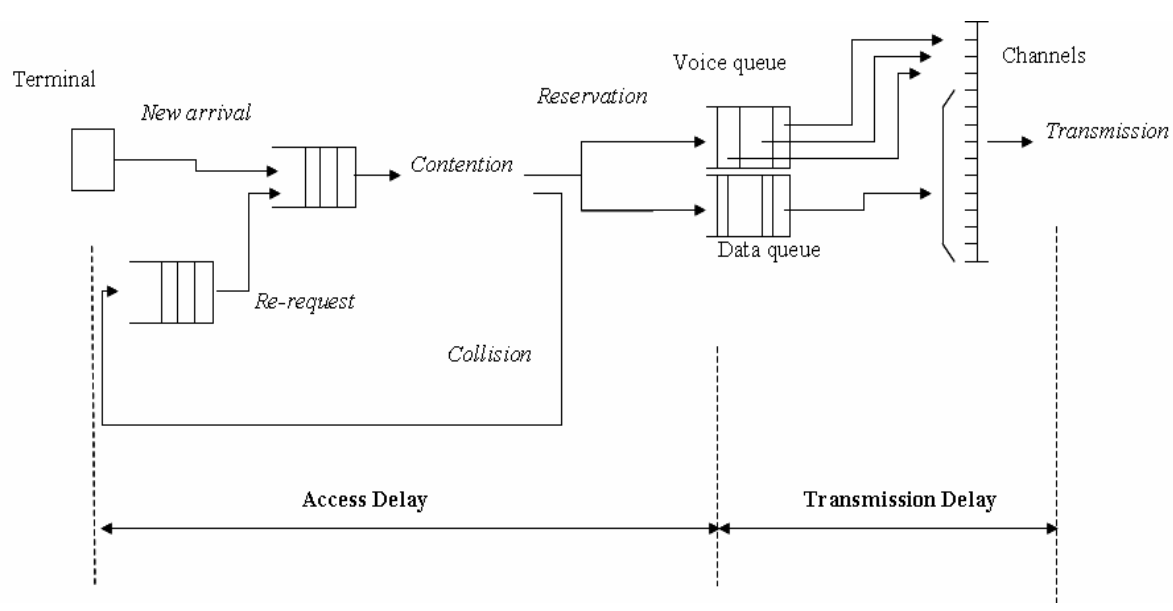

Fig. 1. Transmission procedure

New calls arrive from infinite number of MTs forming a Poisson process with rate $\lambda$ to the system. The time is divided into slots which are grouped into frames of fixed size. A frame is divided into two groups of multiple slots, request slots for reservation of channel and transmission slots for transmission of the actual information. The numbers of request slots and transmission slots in a frame are $V$ and $T$, respectively.

The types of calls are divided into real time call traffic such as voices, and non-real time call traffic like data. Newly arrived call is assumed to be real time calls and nonreal time calls with probabilities $\alpha$ and $\beta$, respectively $(\alpha+\beta=1)$. For notational simplicity, we use the terms voice call or call for real-time and data call or call for non-real time. Let the numbers of packets in a voice call and a data call are geometrically distributed with means $1 / \varepsilon$ and $1 / \delta$, respectively. It is essential that a priority is given to the voice traffic, but an effort is made to accommodate data traffic, whenever possible. When a call arrives at the cell, it waits until the beginning of the next frame 
and randomly accesses one of the request slots to reserve a channel for transmission. If the call succeeds in the reservation, then a channel is allocated in any cell. If, however, two or more call contend for the same request slot, then a collision occurs and none of the calls can reserve the request slot.

The call which fails to get a request slot retries under the BEB algorithm: whenever a call is involved in a collision and if it was the $\mathrm{b}^{\text {th }}(b=0,1, \cdots, 15)$ collision, then it selects one of the next $2^{i}$ frames with probability $1 / 2^{i}$ and attempts the reservation again, where $i=\min (b, 10)$. If a call collides 16 times, then it fails to transmit and is dropped. Those calls reserved slots then enter the queues and transmit their packets according to the proper scheduling method.

\section{Analytic Model}

We obtain the SSD of the number of calls at the beginning of the frame. Let $A_{n}$ be the number of new calls arrived during the nth frame and $N_{n}$ be the total number of calls waiting in the system at the beginning of the nth frame. Also, denote $J_{n}$ by the number of calls which successfully reserve a request slot at the nth frame. Then it can be shown that

$$
N_{n+1}=\left\{\begin{array}{cc}
N_{n}-J_{n}+A_{n}, & N_{n} \geq 1, \\
A_{n}, & N_{n}=0,
\end{array}\right.
$$

and $\left\{N_{n}, n \geq 1\right\}$ is a Markov chain. Let us denote $a_{j}, j=0,1,2, \cdots$ by the Steady State Distribution (SSD) of $A_{n}$, where $a_{j}=\operatorname{Pr}\left(A_{n}=j\right)=e^{-\lambda d}(\lambda d)^{j} / j !, \quad j=0,1,2, \cdots$. Let us introduce a random variable $Y_{n}$ that is the number of calls which actually participate in the contention at the $n^{\text {th }}$ frame. Then for $J(y, k)=\lim _{n \rightarrow \infty} \operatorname{Pr}\left(J_{n}=k \mid Y_{n}=y\right)$ and $Y(i, y)=\lim _{n=\infty} \operatorname{Pr}\left(Y_{n}=y \mid N_{n}=i\right)$, from [5],

$$
J(y, k)=\frac{(-1)^{k} V ! y !}{V^{y} k !} \sum_{m=k}^{\min (V, y)}(-1)^{m} \frac{(V-m)^{y-m}}{(m-k) !(V-m) !(y-m) !}
$$

for $0 \leq k \leq \min (V, y)$ and

$$
Y(i, y)=\left(\begin{array}{c}
i \\
y
\end{array}\right) r^{y}(1-r)^{i-y}, \quad y=0,1, \cdots, i,
$$

where $r$ is the probability that an arbitrary call participates in the contention. We derive this probability by conditioning the number of collisions that an arbitrary message waiting in the system has experienced as following:

$$
r=\left(\frac{1-\left(\gamma_{c} / 2\right)^{11}}{1-\left(\gamma_{c} / 2\right)}\right) \frac{1-\gamma_{c}}{1-\gamma_{c}^{16}}+\left(\gamma_{c} / 2\right)^{10} \frac{\gamma_{c}\left(1-\gamma_{c}^{5}\right)}{1-\gamma_{c}^{16}},
$$


where $\gamma_{c}$ is the probability that an arbitrarily chosen (tagged) call experiences a collision when it contends for a request slot, which will be derived in the next subsection. Now we can calculate the one-step transition probability $p_{i j}=\operatorname{Pr}\left(N_{n+1}=j \mid N_{n}=i\right)$ as given below:

$$
p_{i j}=\sum_{k=\max (0, i-j)}^{\min (i, V)} a_{j-i+k} \sum_{y=0}^{i} J(y, k) Y(i, y)
$$

for $i \geq 1$ and $p_{0 j}=a_{j}$. The Steady State Probability Distribution (SSPD) $\pi_{j} \equiv \operatorname{Pr}(N=j)=\lim _{n \rightarrow \infty} \operatorname{Pr}\left(N_{n}=j\right)$ of the number of messages in system at the beginning of the frame can be obtained by solving the steady state equations $\pi_{i}=\sum_{i=0}^{\infty} \pi_{i} p_{i j}$ and $\sum_{i=0}^{\infty} \pi_{i}=1$.

Now, we derive the collision probability, $\gamma_{c}$, that a tagged message experiences a collision given that it actually participates in the contention for a request slot in this subsection. This probability has not been found in an analytic form in the previous studies and we calculate it for the first time in this paper. Let $M$ be the number of calls in the system at the beginning of the frame in which the tagged call is included. It is known that $M$ is differently distributed from $N$ because it contains the tagged call [6]. The Probability Distribution (PD) of $M$ is given by

$$
\operatorname{Pr}(M=m)=\frac{m \pi_{m}}{E(N)}, \quad \text { where } \quad E(N)=\sum_{j=0}^{\infty} j \pi_{j} .
$$

When $y$ messages including the tagged call participate in the contention, the probability that the tagged call collides is $\sum_{i=1}^{y-1}\left(\begin{array}{c}y-1 \\ i\end{array}\right)\left(\frac{1}{V}\right)^{i}\left(1-\frac{1}{V}\right)^{y-1-i}$. Therefore, we have the following:

$$
\begin{aligned}
\gamma_{c} & =\sum_{m=2}^{\infty} \sum_{y=2}^{m} \sum_{i=1}^{y-1}\left(\begin{array}{c}
y-1 \\
i
\end{array}\right)\left(\frac{1}{V}\right)^{i}\left(1-\frac{1}{V}\right)^{y-1-i} \cdot Y(m, y) \cdot \operatorname{Pr}(M=m) \\
& =\sum_{m=2}^{\infty}\left\{1-\frac{V}{V-1}\left(1-\frac{r}{V}\right)^{m-1}+\frac{1}{V-1}(1-r)^{m}\right\} \cdot m \pi_{m} / \sum_{j=0}^{\infty} j \pi_{j} .
\end{aligned}
$$

Note that the probability that a call is eventually blocked is $\gamma_{c}^{16}$. In order to obtain $\gamma_{c}$ in Eq. (7), we need $\pi_{j}$ but in turn $\gamma_{c}$ should be given to obtain $\pi_{j}$. So we perform a recursive computation, i.e., we initially set $\gamma_{c}$ to be an arbitrary value between 0 and 1 and compute $\pi_{j}, j=0,1,2, \cdots$. Then with this $\pi_{j}$, we update $\gamma_{c}$ using the Eq. (8) and this updated $\gamma_{c}$ is utilized to update $\pi_{j}$ again. This recursive computation continues until both values converge. 


\section{Expected Access Delay}

Now we derive the expected medium access delay of a call which is defined as the time from the moment that a call arrives at the system to the moment that it successfully reserves a request slot. It can be obtained by counting the number of frames from which a newly arrived call contends for a slot for the first time until it successfully reserves a slot. If a call reserves in the first trial with no collision (i.e., $b=0$ ), then it experiences, on average, $3 / 2$ frame length's delay, which is the sum of $1 / 2$ frame length (average length from the call's arrival epoch to the beginning of the next frame) and 1 frame length. Suppose a call collides exactly $b$ times then it selects one of $2^{n}$ states with equal probability and thus the average number of frames it has spent in the system is $\frac{1}{2}+\sum_{i=0}^{b-1} 2^{i}+\frac{1}{2^{b}} \sum_{j=0}^{2^{b}} j$. In the same manner, if $11 \leq b \leq 15$, the average delay is $\frac{1}{2}+\sum_{i=0}^{10} 2^{i}+(b-11) \cdot 2^{10}+\frac{1}{2^{10}} \sum_{j=0}^{2^{10}} j \cdot$ We obtain the expected access delay, $E\left(D_{\text {Access }}\right)$, in frames, as the following:

$$
\begin{aligned}
& E\left(D_{\text {Access }}\right)=\sum_{b=0}^{15} E\left(D_{\text {Access }} \mid b \text { collisions }\right) \operatorname{Pr}(b \text { collisions })=\frac{1}{2}+\frac{1-\gamma_{c}}{1-\gamma_{c}{ }^{16}} \times \\
& \left(\begin{array}{c}
1+2.5 \gamma_{c}+5.5 \gamma_{c}{ }^{2}+11.5 \gamma_{c}{ }^{3}+23.5 \gamma_{c}{ }^{4}+47.5 \gamma_{c}{ }^{5}+95.5 \gamma_{c}{ }^{6}+191.5 \gamma_{c}{ }^{7}+383.5 \gamma_{c}{ }^{8}+767.5 \gamma_{c}{ }^{9} \\
+1535.5 \gamma_{c}{ }^{10}+2559.5 \gamma_{c}{ }^{11}+3583.5 \gamma_{c}{ }^{12}+4607.5 \gamma_{c}{ }^{13}+5631.5 \gamma_{c}{ }^{14}+6655.5 \gamma_{c}{ }^{15}
\end{array}\right) .
\end{aligned}
$$

The PD of the number, $Z$, of calls which reserve request slots successfully in a frame can be obtained by conditioning $Y$ and $N$ as following:

$$
\operatorname{Pr}(Z=x)=\sum_{n=0}^{\infty} \sum_{y=0}^{n} J(y, x) Y(n, y) \pi_{n} .
$$

The expected number $E(Z)$ is given by

$$
E(Z)=\sum_{n=0}^{\infty} \sum_{y=0}^{n} E(Z \mid Y=y, N=n) \operatorname{Pr}(Y=y \mid N=n) \pi_{n}=\sum_{n=0}^{\infty} n r\left(1-\frac{r}{V}\right)^{n-1} \pi_{n},
$$

where the second equality comes from the Eq. [6].

\section{Transmission Delay of Voice/ Data Calls}

Now we consider the expected transmission delay of a call, which is the time duration elapsed between a call succeeds in the contention and it is successfully transmitted. A fundamental requirement in the voice communication is prompt delivery of information. In our paper, we put a buffer of size $B$ for voice calls, with which one can adjust the allowable delay of voice call until its successful transmission. For example, if a longer delay for voice is allowed with low packet dropping probability then we make $\mathrm{B}$ bigger. This is in contrast to the data messages which respond to congestion and transmission impairments by delaying packets in queue. So we assume the buffer size for data calls is unlimited. 
Each voice call uses one slot in a frame and transmits one packet per frame, which accommodates the real time transmission requirement on the voice calls. All $T$ transmission slots are available for voice transmission, so maximum $T$ voice calls can transmit simultaneously, while the data calls can transmit their packets only when there are less than $T$ slots occupied by the voice calls. Even during a data call is sending its packets, if an arriving voice message finds no idle slots, then the data call interrupts its transmission and hand over one slot to the voice message. That is, the voice messages are preemptive. The interrupted data call resumes its transmission whenever there are any slots available. We analyze transmission delays for each type of calls in the sequel.

Notice that the transmission of voice calls is independent of the transmission of data calls. Denote $K_{n}$ by the number of voice calls which succeed in the contention during the $n^{\text {th }}$ frame and newly join at the voice transmission queue. Since a call is voice call with probability $\alpha$, the PD of $K_{n}$ can be obtained from the Eq. (9) as following:

$$
\operatorname{Pr}\left(K_{n}=l\right) \equiv k_{l}=\sum_{x=l}^{\infty} \operatorname{Pr}(Z=x)\left(\begin{array}{l}
x \\
l
\end{array}\right) \alpha^{l}(1-\alpha)^{x-l}, \quad l=0,1,2, \cdots
$$

Then $X_{n}$, the number of voice calls in the system at the beginning of the $n^{\text {th }}$ frame has the relationship $X_{n+1}=X_{n}-\xi\left(X_{n}\right)+K_{n}$, where $\xi_{n}\left(X_{n}\right)$ is the number of voice calls which complete the transmission at the $n$th frame given $X_{n}$. The PD of $\xi_{n}\left(X_{n}\right)$ when $X_{n}=i$, assuming the number of packets in a voice call is geometric with mean $1 / \varepsilon$, is given by

$$
\operatorname{Pr}\left(\xi_{n}(i)=k\right)=\left(\begin{array}{c}
\min (i, T) \\
k
\end{array}\right)(1-\varepsilon)^{\min (i, T)-k} \varepsilon^{k} \equiv \xi_{n}(\min (i, T), k),
$$

for $k=0,1, \cdots, \min (i, T)$, Let $\lim _{n \rightarrow \infty} X_{n}=X, \lim _{n \rightarrow \infty} K_{n}=K, \lim _{n \rightarrow \infty} \xi_{n}=\xi$. It can be shown that $\left\{X_{n}, n \geq 1\right\}$ is a Markov chain. Then we can obtain the one-step transition probabilities $q_{i j}$ of this chain as following:

$$
q_{i j}=\left\{\begin{array}{cc}
\sum_{l=0}^{j} k_{l} \xi(\min (i, T), \min (i, T)-j+l), & j=0,1, \cdots, T+B-1, \\
\sum_{l=0}^{\infty} k_{l} \xi(\min (i, T), \min (i, T)-j+l), & j=T+B,
\end{array}\right.
$$

for all $i=0,1, \cdots, T+B$ and $\xi(i, k)=\xi(i, i)$ if $k \geq i$ and $\xi(i, k)=0$ if $k<0$. Now the SSPD, $\eta=\left(\eta_{0}, \eta_{1}, \cdots, \eta_{T+B}\right)$, where $\eta_{j}=\operatorname{Pr}(X=j)$, of the number of voice calls to be transmitted in the system at the beginning of the frame is given by solving the equations:

$$
\eta_{j}=\sum_{i=0}^{T+B} \eta_{i} q_{i j}, \sum_{j=0}^{T+B} \eta_{j}=1
$$

The expected transmission delay of voice message in frames is 


$$
E\left(D_{\text {voice }}\right)=E(X) / E(K)
$$

by applying the well-known Little's result [6].

As explained before, the data call can transmit its packets whenever there are slots available. Therefore, the PD of the number of packets waiting to be transmitted in the queue depends on how many slots are currently being held by voice transmission. Denote $U_{n}$ by the number of data calls which succeed in the contention during the $n^{\text {th }}$ frame and newly join at the data transmission queue. A call is data message with probability $\beta$, and thus

$$
\operatorname{Pr}\left(U_{n}=l\right) \equiv u_{l}=\sum_{x=l}^{\infty} \operatorname{Pr}(Z=x)\left(\begin{array}{c}
x \\
l
\end{array}\right)(1-\beta)^{x-l} \beta^{l},
$$

and the $\mathrm{PD}, w_{j}, j \geq 0$, of the number of data packets arrived during a frame is:

$$
w_{j}=\sum_{l=1}^{\infty} u_{l}\left(\begin{array}{c}
j-1 \\
l-1
\end{array}\right) \delta^{l}(1-\delta)^{k-l}, j \geq 1,
$$

and $w_{0}=u_{0}$. Let $k$ be the number of slots which are occupied for voice transmission.

By means of the probabilities $w_{j}$, we can find the steady state conditional PD, $v^{(k)}=\left(v_{0}{ }^{(k)}, v_{1}^{(k)}, v_{2}^{(k)}, \cdots\right)$, of the number of data packets in the system at the beginning of the frame, when $k$ voice calls are transmitting, by solving the following steady-state equations:

$$
\begin{aligned}
& v_{0}^{(k)}=w_{0}\left(\sum_{i=0}^{T-k} v_{i}^{(k)}\right), \\
& v_{j}^{(k)}=w_{j}\left(\sum_{i=0}^{T-k} v_{i}^{(k)}\right)+\left(\sum_{m=1}^{j} v_{T-k+m}^{(k)} w_{j-m}\right), j \geq 1, \text { and } \sum_{j=0}^{\infty} v_{j}^{(k)}=1 .
\end{aligned}
$$

Finally, the unconditional PD $v=\left(v_{0}, v_{1}, v_{2}, \cdots\right)$ is:

$$
v_{j}=\sum_{k=0}^{\infty} v_{j}^{(k)} \eta_{k}, j=0,1,2, \cdots
$$

Now we can calculate the expected number of data packets at the beginning of the frame, that is $L_{D}=\sum_{j=0}^{\infty} j v_{j}$. Then $(T-E(X)) /\left(\delta \cdot L_{D}\right)$ is the expected number of frames to transmit one data call, which gives the transmission delay in frames of a data call.

\section{Numerical Results}

In numerical study to verify our model, we compute the expected (i.e., access and transmission) delays for voice calls and data calls using the parameters $V=30, T=95, B=10, \lambda=5, \alpha=0.3$ and the expected number of packets of a data call is set to be 1000 (i.e., $\delta=0.001$ ). Expected delays are calculated as the expected 
number of packets in a voice call (i.e., $1 / \varepsilon$ ) varies. The same was found using simulations as well.

The plots in Fig. 2 show close agreement between analytic results and the simulation, especially in the voice call, thus validating the analysis. As for the data call, however, analytical results tend to overestimate than the simulation but are still within the confidence intervals. This is because we first derived the probability distribution of the number of data packets in the system under the condition that there are $k$ voice calls transmitting, and then unconditioned it. This is based on the assumption that the number of data packets reaches steady state before $k$ changes.
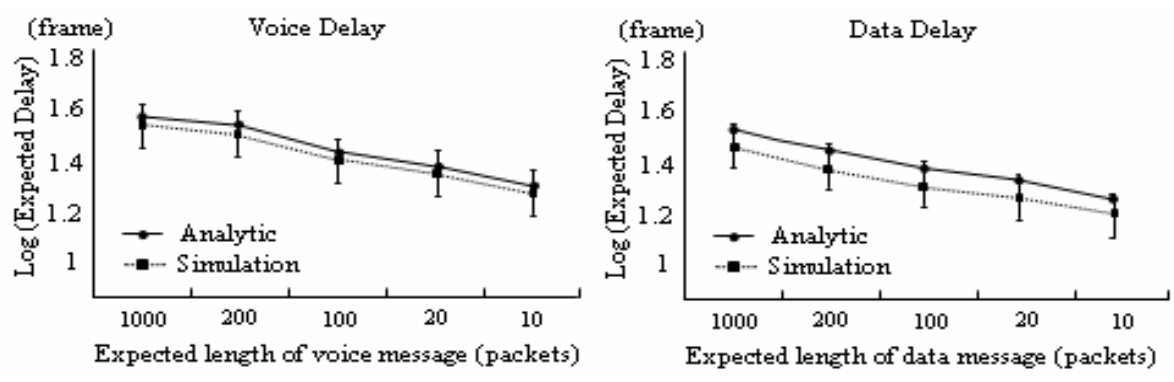

Fig. 2. Expected delays

\section{Conclusion}

In this paper we considered the analytic model of the BEB policy, which is a coll ision resolution algorithm often adopted in the random access packet networks. We obtain the SSD of the number of messages waiting in the system, which is utilized to get the probability that a tagged call experiences a collision given that it actually participates in the contention for a request slot in a frame, which has never been investigated in the literature. With these, the expected access and transmission delay of frames that a message experiences from its arrival to the system until the successful transmission are found analytically. A numerical results from the analytic model is provided. It shows that our proposed analytic model gave an excellent agreement with the simulation.

\section{Acknowledgements}

This work was supported by Fund of Howon University, 2006.

\section{References}

1. Yung-Fang Chen, Chih-Peng Li.: Performance Evaluation of the Quasi-FIFO Back-off Scheme Wireless Access Networks. IEEE VTC. Vol. 2 , 2003

2. Yang Yang, and Tak-Shing Peter Yum.: Delay Distributions of Slotted ALOHA and CSMA. IEEE Trans. on Comm., Vol.51, 2003 
3. P. Chatzimisios, V. Vitsas and A. C. Boucouvalas.: Throughput and Delay Analysis of IEEE 802.11 Protocol. Networked Appliances, Liverpool. Proceedings, 2002 pp. 168 - 174

4. B. J. Kwak, N. O. Song, and L. E. Miller.: Analysis of the Stability and Performance of Exponential Backoff. IEEE WCNC, Vol. 3, 2003

5. W. Szpankowski.: Analysis and stability considerations in a reservation multiaccess system. IEEE Trans. on Comm., Vol. com-31, 1983

6. L. Kleinrock.: Queueing system, Vol. 1. Theory. John Wiley \& Sons, 1975 